\title{
Differences in Brain Activity for Temporal Lobe Epilepsy Patients and Healthy Subjects: Findings from Resting- state fMRI
}

\author{
Jan Mohamed $\mathrm{S}^{1}$, Mohd Amin Rebuan $\mathrm{H}^{2}$, Abd Hamid $\mathrm{Al}^{3}$, Yusoff $\mathrm{AN}^{4}$ \\ ${ }^{1}$ Faculty of Health Sciences, Universiti Sultan Zainal Abidin, Terengganu, Malaysia, \\ ${ }^{2}$ Faculty of Medicine, Universiti Sultan Zainal Abidin, Terengganu, Malaysia, \\ ${ }^{3}$ Department of Neurosciences, School of Medical Sciences, Universiti Sains Malaysia, Kelantan, Malaysia \\ ${ }^{4}$ Centre for Diagnostic, Therapeutic and Investigative Studies, Faculty of Health Science, Universiti Kebangsaan Malaysia, Kuala Lumpur, Malaysia
}

Keywords

temporal lobe epilepsy, brain lateralisation, precuneus, supramarginal, rsfMRI,

functional connectivity

Corresponding Author

Dr Husbani Mohd Amin Rebuan, Faculty

of Medicine, Universiti Sultan Zainal

Abidin, Terengganu, Malaysia

Tel No: $+013-938778$

Email : husbanimar@unisza.edu.my

Received: 19th Sept 2021; Accepted: 25th October 2021

Doi: https://doi.org/10.31436/imjm.v21i1

\section{ABSTRACT}

INTRODUCTION: Temporal lobe epilepsy (TLE) is a chronic disorder of the nervous system with focal seizures that may spread to other brain regions. Present knowledge regarding the spread of seizure is scarce. This study investigated activity in brain regions outside temporal lobe of TLE patient (TLEP) that may be affected by the spread. The findings were compared with healthy subject (HS). MATERIALS AND METHODS: Resting-state functional MRI (rsfMRI) were performed on 14 TLEPs and 14 HSs. Spatial activation, laterality index (LI) and functional connectivity (FC) involving several brain regions were analysed. RESULT: Bilateral precuneus (PRE) and supramarginal gyrus $(\mathrm{SMG})$ which were activated ( puncorr. $<0.001$ ) outside the temporal lobe were chosen for analysis. In the left and right hemispheres, two-way analysis of variance (ANOVA) showed a significant difference between PRE and SMG activation $(p<0.001)$ but not between TLEP and HS ( $\mathrm{p}>0.001)$. SMG was found to be moderately right-lateralised in both TLEP and HS with LI $=-0.309$ and -0.125 , respectively. For PRE, HS showed moderate left lateralisation ( $\mathrm{LI}=0.121$ ), while TLEP showed weak right lateralisation $(\mathrm{LI}=-0.002)$. FC results revealed that the activity in PRE and SMG changed over time for HS and TLEP but only in the left hemisphere. CONCLUSION: Although resting-state activations in the two selected brain areas outside the temporal lobe in both hemispheres were incomparable between TLEP and HS, evidences from LI and FC analyses suggested anomalies especially in the left hemisphere that could be due to the spread of seizure.

\section{INTRODUCTION}

Temporal lobe is a part of the brain that processes emotion and regulates short-term memory. ${ }^{1}$ Previous functional magnetic resonance imaging (fMRI) studies have reported that temporal lobe epilepsy (TLE) affects brain regions outside the temporal lobe and is associated with abnormal brain networks. ${ }^{2}$ Patient may have odd feelings such as euphoria, deja vu and fear. ${ }^{3}$ The feelings may stem from anatomical defects or scarring of the temporal lobe 4 but the underlying cause is unknown. Temporal lobe seizures can be treated with medication and surgery for those who do not respond to medication. ${ }^{5}$

Restricted resections to the pathogenic tissue, stereotactic laser, radiosurgery are all associated with better cognitive outcomes. ${ }^{6}$ However, the level of resection is limited by the potential for cognitive deficits. Intracarotid amobarbital procedure (IAP) also known as WADA test is conducted to predict memory impairment from TLE surgery, but the invasiveness of this technique has been debated by many. ${ }^{6-8}$ The fMRI is a non-invasive technique that can potentially replace the WADA test as a presurgical tool to evaluate brain functions in patients with TLE. 6,9

The fMRI has the potential to predict possible deficits in language, visual, motor and sensory functions that would arise as a result of surgical intervention or post operation. ${ }^{10}$ Findings from rsfMRI studies on patients 
with TLE e.g. functional connectivity (FC) indicated that rsfMRI was able to provide information for distinguishing TLE with mesial temporal sclerosis from TLE without mesial temporal sclerosis. ${ }^{11}$

According to Goldstein at al. ${ }^{12}$ there is a tendency for some brain functions to be specialized to a specific hemisphere of the brain, known as brain lateralisation. This functional specialisation feature of the brain may be affected by seizures in an epilepsy episode. A common way of measuring changes in lateralisation is by calculating the laterality index (LI). LI is used to assess hemispheric dominance in various tasks, such as language, cognitive and sensory. ${ }^{13}$ Knowledge of brain lateralisation in patients with TLE is important in determining the clinical outcomes. For example, left lateralisation correlates with peri-ictal aphasia in patients with left TLE. ${ }^{14}$

This study aimed to investigate brain activities of age and gender matched temporal lobe epilepsy patient (TLEP) and healthy subject (HS). Due to the possible effects of TLE on brain activity, it is hypothesised that if there is a spread of TLE seizure to the areas outside the temporal lobe, we will see changes in brain activation, LI and FC in TLEP compared to HS.

\section{MATERIALS AND METHODS}

\section{Subjects}

Fourteen HSs (11 females, 3 males) and 14 TLEPs (11 females, 3 males) matched by age and gender participated in this study. All HSs were free of medical illness and recruited from those who accompanied their relatives at the polyclinic and also among the staff of Hospital Universiti Sains Malaysia (HUSM). The clinical diagnosis of TLE was made by an attending neurologist based on clinical semiology of seizures and video-EEG monitoring. All TLEPs are already on treatment. All HSs and TLEPs agreed to participate in this study by signing an informed consent form. This study was approved by the institutional ethics committee (IEC) USM/ JEPeM/16050175. Subjects with major brain abnormalities and psychiatric disorders were excluded.

\section{Resting-state fMRI Scans}

Resting-state fMRI BOLD imaging protocol was performed using a 3-T Phillips Achieva MRI scanner equipped with a 32-channel head and neck system at Radiology Department, HUSM. The echo planar imaging (EPI) parameters for acquiring functional T2* weighted images were echo time $(\mathrm{TE})=33 \mathrm{~ms}$, repetition time (TR) $=1.7 \mathrm{~s}$, flip angle $\left({ }^{\circ}\right)=75^{\circ}$, slice thickness $=4 \mathrm{~mm}$, slice gap $=0 \mathrm{~mm}$, field of view $(\mathrm{FOV})=192 \times 192 \mathrm{~mm}$, matrix size $=64 \times 64$, voxel size $=2 \times 2 \times 4 \mathrm{~mm}$, number of scans $=250$ and total imaging time $=425 \mathrm{~s}$ (7.1 mins). The subjects were instructed not to move their heads during the scan and to passively focus on a fixation point " $x$ " symbol throughout the session.

\section{Data Analysis}

\section{Spatial Processing}

A total of 247 functional images were analyzed using Statistical Parametric Mapping (SPM12), Institute of Neurology (ION), University College London (UCL), UK-www.fil.ion.ucl.ac.uk/spm/) which runs on MATLAB R2016b platform (The Mathworks, Inc. USA). The functional images were preprocessed as described elsewhere. ${ }^{15,16}$

\section{Group activation}

The functional images were then entered into group analysis to find voxels that survived the uncorrected significance level of 0.001 by means of fixed-effects analysis (FFX). An anatomically opened hypothesis was conducted to find voxels representing areas in the brain that survived the threshold. Two significant brain areas with highest number of activated voxels (NOV) were chosen from this analysis.

\section{Modelling of Low Frequency Fluctuation}

Human brain has been found to exhibit low-frequency signal (LFF) when at rest. This LFF is in the range of 0.01 to $0.08 \mathrm{~Hz}^{17}$ which is associated with internally and 
externally oriented consciousness. ${ }^{18}$ In this study, all functional images from $14 \mathrm{HSs}$ and 14 TLEPs were entered into a general linear model (GLM) (Fig. 1(a)). Using MATLAB-based WFU Pick Atlas toolbox (Wake Forest University, North Carolina, USA), the two significantly activated brain areas in the left and right hemispheres were obtained and their NOV, statistics and maximum intensity coordinates were recorded. This was performed for individual HS and TLEP at $\mathrm{p}<0.001$, uncorrected for multiple comparisons. The NOV was compared between the left and right hemispheres, and HS and TLEP using Statistical Packages for Social Sciences (SPSS) Version 25.0.

\section{Laterality index}

The laterality index (LI) was calculated to examine hemispheric dominance in TLE and HSs using the NOV mentioned above, as described in Othman et al. (2019). ${ }^{19}$ The threshold of LI (LITH) used in this study was 0.2 from which a region is classified as left hemisphere dominant if LI $>$ LITH, symmetry if LITH $\leq$ LI $\leq$ LITH and right hemisphere dominant if LI $<$ LITH. The LI values for each region were initially obtained from each subject and patient.

\section{Signal Extraction}

A general linear model (GLM) containing time corrected, realigned, normalized and smoothed images was redefined for each particular subject and a third design matrix was constructed (Figure 1(b)). This design matrix was then estimated and used to extract time series signals from cerebrospinal fluid (CSF) and white matter (WM) centered at $(0,-40,-5)$ and $(0,-24,-33)$ of a $6-\mathrm{mm}$ radius volume of interest (node), respectively.

The design matrix in Figure 1(c) was then used to extract the signals from the $8-\mathrm{mm}$ radius ROI1 and ROI2. The time series signals extracted from ROI1 and ROI2 were incorporated into another design matrix, together with the signals extracted from CSF and WM, six realigned parameters and global. This newly constructed design matrix as shown in Figure 1(d) was used to generate activation for ROIs for each subject by the $t$-contrast ( $t$ test) find the effects on these nodes while the brain was at rest. Group activation was then produced using onesample $t$-test at the second level, in the random-effects framework, corrected for multiple comparisons $(p<0.05)$, testing the effects on no activation.

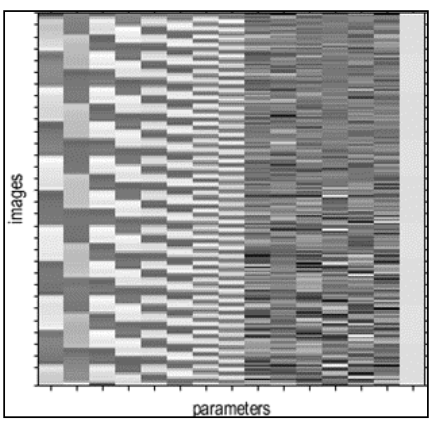

Figure 1 (a) Design matrix containing regressors to model low frequency fluctuation (column 1 to 8 ) and subject's movement (column 9 to 14 ).

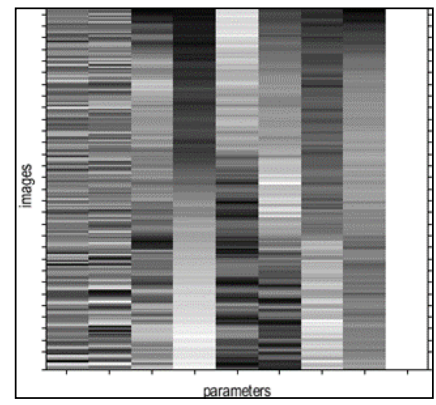

Figure 1(c) Design matrix containing random fluctuations from WM and CSF, which was used to obtain random fluctuations from left ROI1, right ROI1, left ROI2 and right ROI2.

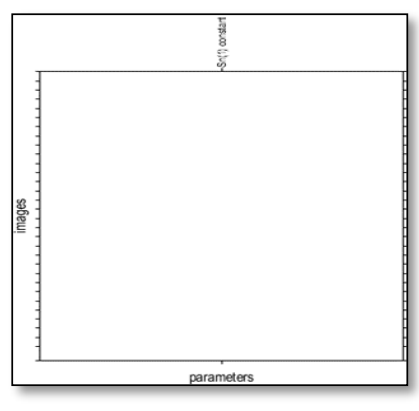

Figure 1(b) Design matrix used for obtaining endogenous random fluctuations from white matter (WM) and cerebrospinal fluid (CSF).

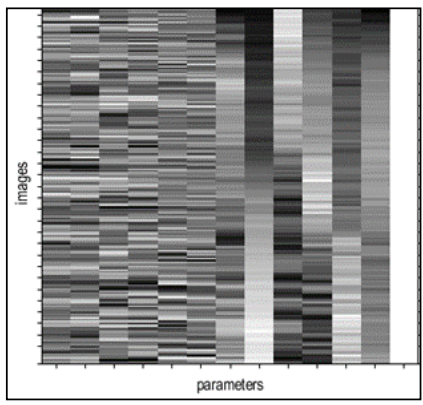

Figure 1(d) Design matrix used to generate activation for ROIs for each subject.

\section{Correlation analysis}

The relationship between the signals extracted from the two regions was also investigated through correlation analysis (SPSS) to determine the existence of temporal relationship between the activity in the activated areas for all subjects. The slopes $(\mathrm{M})$ obtained from both groups of subjects e.g. $\mathrm{M}_{\text {roi1-roi2 }}$ or $\mathrm{M}_{\text {roi2-roi1 }}$ were averaged and compared using independent t-test. Comparisons on the slopes were also made between the two hemispheres. Only intra hemispheric connectivity was considered in this study. 


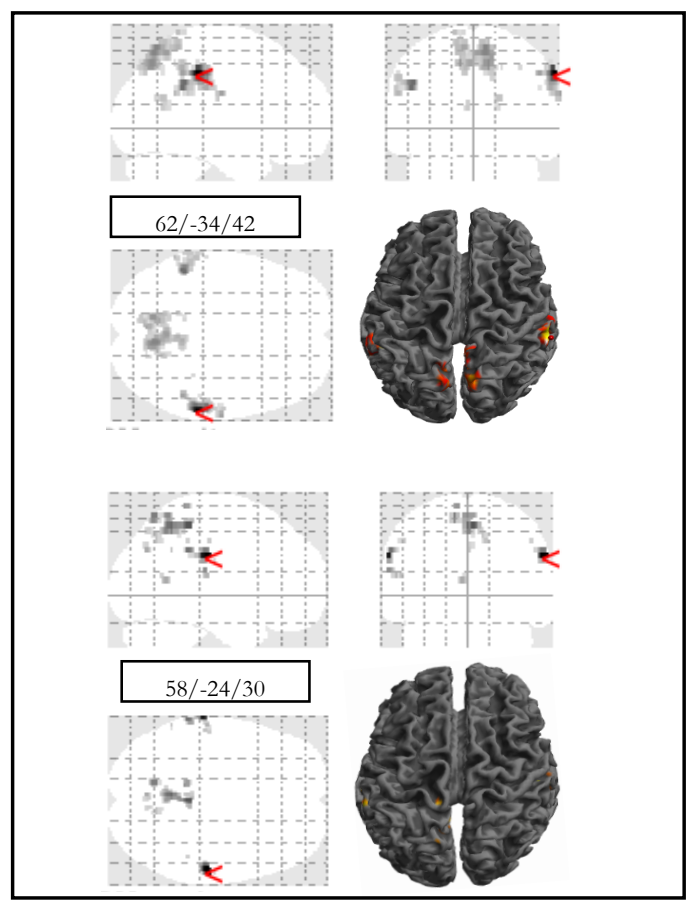

Figure 2 Brain activation ( $\left.\phi_{\text {uncorr. }}<0.001\right)$ from group FFX obtained from (a) healthy subjects and (b) TLE patients. Coordinates of maximum intensity are shown on each figure and indicated by the red arrow heads.

Table 1 contains NOV for left and right PRE and SMG in HS and TLEP when their brains were at rest. The NOV for both regions was obtained from single subject activation at uncorrected significant level (puncorr. $=0.001$ ). Inconsistent NOV for both regions in the left and right hemispheres can be seen across HS and TLEP. Two-way ANOVA tested on brain activation in the right hemisphere showed significant effect of factor on the region $(p<0.001)$ but not on the group $(p=0.776)$. There were no significant effects of factor on group $(p=0.132)$ and on region $(p=0.132)$ in the left hemisphere.

Furthermore, there was no evidence of significant interactions between the factors. Comparison between the mean NOV for left and right SMG using independent -sample Mann-Whitney $U$ test revealed no significant difference $(\mathrm{U}=108.0, \mathrm{p}=0.667$ and $\mathrm{U}=109.5, \mathrm{p}=0.603$ ) between HS and TLEP. Similarly, comparison between the mean NOV for left and right PRE revealed no significant difference $(U=83.5, p=0.511$ and $U=94.5$, $\mathrm{p}=0.874)$ between both groups.
Table 1 Individual subject's activation (NOV) for left and right PRE and SMG in HS and TLEP

\begin{tabular}{llllllllll}
\hline \multicolumn{3}{c}{ NOV } & \multicolumn{7}{c}{ TLEP } \\
& HS & \multicolumn{7}{c}{ R } \\
\hline Subject & Left & Right & Left & Right & Patient & Left & Right & Left & Right \\
No & PRE & PRE & SMG & SMG & No & PRE & PRE & SMG & SMG \\
1 & 76 & 6 & 52 & 0 & 1 & 13 & 45 & 0 & 5 \\
2 & 100 & 71 & 53 & 124 & 2 & 15 & 17 & 11 & 15 \\
3 & 642 & 337 & 89 & 157 & 3 & 30 & 28 & 33 & 32 \\
4 & 198 & 126 & 70 & 48 & 4 & 4 & 1 & 2 & 0 \\
5 & 179 & 110 & 0 & 8 & 5 & 8 & 41 & 0 & 12 \\
6 & 4 & 27 & 0 & 5 & 6 & 89 & 126 & 44 & 9 \\
7 & 68 & 52 & 0 & 0 & 7 & 144 & 175 & 161 & 424 \\
8 & 65 & 158 & 5 & 13 & 8 & 231 & 191 & 58 & 47 \\
9 & 423 & 329 & 77 & 114 & 9 & 48 & 100 & 30 & 37 \\
10 & 361 & 242 & 91 & 105 & 10 & 425 & 227 & 21 & 34 \\
11 & 44 & 77 & 1 & 21 & 11 & 586 & 430 & 129 & 147 \\
12 & 665 & 486 & 71 & 186 & 12 & 90 & 119 & 119 & 160 \\
13 & 118 & 92 & 0 & 15 & 13 & 229 & 64 & 47 & 41 \\
14 & 36 & 9 & 52 & 32 & 14 & 608 & 347 & 87 & 227 \\
\hline Avg. & 213 & 152 & 40 & 59 & Avg. & 180 & 137 & 53 & 85 \\
\hline
\end{tabular}

\section{Laterality Index}

The laterality indices (LI) for PRE and SMG in HS and TLEP during resting state are shown in Table 2(a) and (b), respectively. The average values were indicated at the bottom of the table. The results showed that the LI for SMG in both HS and TLEP was moderately right lateralized (LI $=-0.309$ and -0.125). In contrast, the LI for PRE in HS was moderately left lateralized (LI $=0.121)$, whereas it was weakly right lateralized for TLEP (LI = $0.002)$.

\begin{tabular}{ccc}
\multicolumn{3}{c}{ Table 2 (a) Laterality index for PRE and SMG in healty subjects } \\
\hline Subject No & SMG & PRE \\
\hline S1 & 1.000 & 0.854 \\
S2 & -0.401 & 0.170 \\
S3 & -0.276 & 0.312 \\
S4 & 0.186 & 0.222 \\
S5 & -1.000 & 0.239 \\
S6 & -1.000 & -0.742 \\
S7 & 0.000 & 0.133 \\
S8 & -0.444 & -0.417 \\
S9 & -0.194 & 0.125 \\
S10 & -0.071 & 0.197 \\
S11 & -0.909 & -0.273 \\
S12 & -0.447 & 0.157 \\
S13 & -1.000 & 0.124 \\
S14 & 0.238 & 0.600 \\
\hline Average & -0.309 & 0.121 \\
\hline
\end{tabular}

\section{Functional Connectivity}

Fig. 3 (a) shows the plots of MLSMG-LPRE against MLPRE-LSMG for HS and TLEP. A repeated ANOVA with two factors, group (HS and TLEP) and connection (PRE-SMG and SMG-PRE) showed a significant effect of factor on connection $(\mathrm{F}=13.019, \mathrm{p}=0.001)$ but not on 
Table 2 (b) Laterality index for PRE and SMG in TLEPs

\begin{tabular}{ccc}
\hline Patient No & SMG & PRE \\
\hline S1 $2 ~$ & -1.000 & -0.552 \\
S2 & -0.154 & -0.063 \\
S3 & 0.015 & 0.035 \\
S4 & 1.000 & 0.600 \\
S5 & -1.000 & -0.673 \\
S6 & 0.660 & -0.172 \\
S7 & -0.450 & -0.097 \\
S8 & 0.105 & 0.095 \\
S9 & -0.104 & -0.351 \\
S10 & -0.236 & 0.304 \\
S11 & -0.065 & 0.154 \\
S12 & -0.147 & -0.139 \\
S13 & 0.068 & 0.563 \\
S14 & -0.446 & 0.273 \\
\hline Average & -0.125 & -0.002 \\
\hline
\end{tabular}

group $(\mathrm{F}=2.341, \mathrm{p}=0.132)$. No significant interactions between the factors were evident. Both HS and TLEP showed a similar trend of positive linear relationship between the two slopes but with different behaviors for the two regions in the left hemisphere. In the left hemisphere, for all HSs and TLEPs participated in this study, the rate of change of the response in PRE due to activity in SMG was greater than the rate of change of the response in SMG due to the activity in PRE when the brain was at rest. The rate of change was larger in HS compared to TLEP.

Figure 3(b) depicts a positive linear relationship between MRSMG-RPRE and MRPRE-RSMG for both HS and TLEP. A repeated ANOVA measurement with two factors, group (HS and TLEP) and connection (PRESMG and SMG-PRE) showed a significant effect of factor on the connection $(\mathrm{F}=11.575, \mathrm{p}=0.001)$ but not on the group $(\mathrm{F}=0.082, \mathrm{p}=0.776)$. There were no significant interactions between the factors. The results indicated that both subject groups had a similar trend of positive linear relationship between the two slopes but with different behavior for the two regions in the right hemisphere. A smaller increase in MRPRE-RSMG resulted in a double increase in MRSMG-RPRE. Similar to the left hemisphere, for all HSs and TLEPs participating in this study, the rate of change of the response in PRE due to activity in SMG was greater than the rate of change of the response in SMG due to activity in PRE when the brain was at rest. The rate of change in TLEPs was equal to the rate of change in HS.

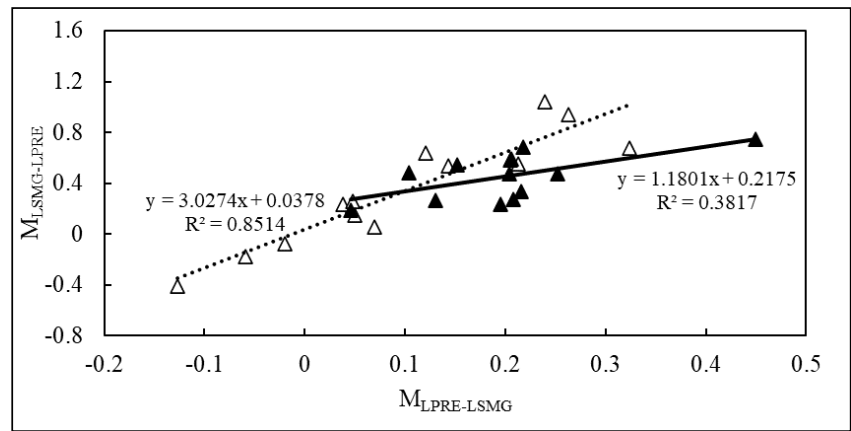

Figure 3(a) Plots of $\mathrm{M}_{\mathrm{LSMG}-\mathrm{LPRE}}$ vs. $\mathrm{M}_{\text {LPRE-LSMG }}$ in left hemisphere for HS (D) and TLEP $(\boldsymbol{\Delta})$

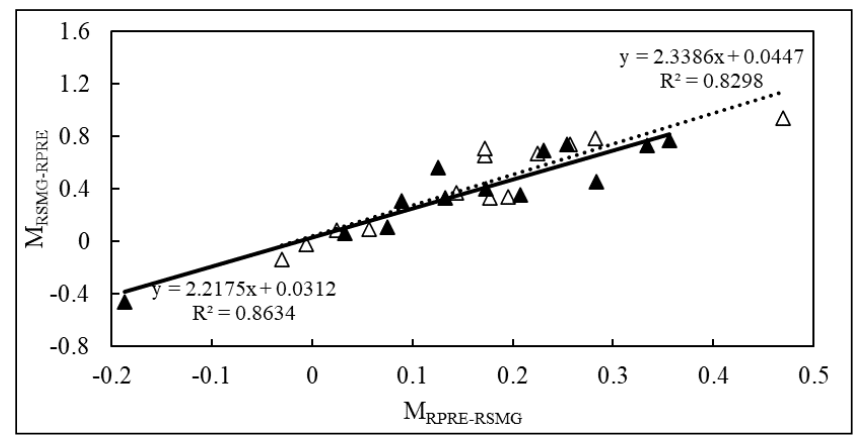

Figure 3(b) Plots of $\mathrm{M}_{\text {RSMG-RPRE }}$ vs. $\mathrm{M}_{\text {RPRE-RSMG }}$ in right hemisphere for HS (D) and TLEP ( $\mathbf{\Delta})$.

Post-hoc tests showed a significant response in the right SMG due to activity in right PRE $(\mathrm{p}<0.05)$ and significant response in the right PRE due to activity in the right SMG $(\mathrm{p}<0.05)$ for both HS and TLEP. Similar results were obtained for the left hemisphere regions. The results indicated that activations in PRE and SMG were not influenced by pathological conditions in the temporal lobes but were influenced by activity in their respective regions. No significant interactions between the factors were evident.

\section{DISCUSSION AND CONCLUSION}

A significant group activations in both PRE and SMG in both HS and TLEP when the brain was at rest were found in this study using fixed-effects analysis on fMRI data but with a lower significant level $\left(p_{\text {uncorr }}<0.001\right)$. Nevertheless, the clusters of activation were significant at the set and cluster levels $\left(p_{\text {corr. }}=0.05\right)$. In a previous study performed on multiple sclerosis patients with passive hand movements ${ }^{22}$, it showed that similar statistical methods of inferences were reported from which significant clusters of activation $\left(p_{\text {corr }}<0.05\right)$ were considered from 
uncorrected activation maps ( $\left.p_{\text {uncorr }}<0.001\right)$. A higher number of activated voxels in PRE and SMG exhibited by HS compared to TLEPs is an indication of changes in brain activation that typically occur in diseased brain. ${ }^{23}$ However, both groups had similar highest intensity point that occurred in the same region of the right hemisphere SMG. In our previous effective connectivity (EC) study between PRE and SMG 24, it was found that the information transfer from PRE to SMG occurred at a higher rate (SMG received more information) which would indirectly support the current findings. Since NOV was higher for PRE than SMG for both HS and TLEP, the role of PRE as a DMN hub would explain this difference. As more areas are connected to a node in a network, greater spatial activation may be observed. ${ }^{25}$

In consistent with the group FFX results on spatial activation as mentioned above, it can be postulated that being an important region in the $\mathrm{DMN}$ has resulted in higher average NOV for PRE compared to SMG for both HS and TLEP groups in the hemisphere as obtained from individual analysis. This may be true as PRE has always been the central hub of the DMN. ${ }^{23}$ However, the insignificant differences obtained from comparisons between brain activation and HS and TLEP for the four regions using individual data may be due to the variability of spatial activation within the group (Table 1). This could also mean that activation in PRE and SMG is not influenced by pathological conditions in the temporal lobes, at least in in the context of this study, when the subjects' brains are at rest.

Right lateralisation of SMG in healthy brain and brain with TLE indicated that SMG was not affected by brain disorders in the temporal lobe. The LI results for SMG corresponded to the results for individual brain activation as above mentioned. However, TLE has caused the PRE to change its lateralisation to the right hemisphere resulting in a balanced lateralisation state. A slight change in brain lateralisation observed in TLEP compared to HS was also due the majority of TLEPs reaching a stable state with on-going treatment. Previous fMRI study has shown sufficient reliability of resting state FC to predict the degree of hemispheric dominance in HS and TLEP. ${ }^{26}$ Dietz et al. ${ }^{16}$ stated that LI is a valuable tool in fMRI research especially to people with post-stroke aphasia. However, there were inconsistent consideration in cases where lesions had overlapped with ROI. ${ }^{27}$ Since all of subjects in this study were free from any pathological lesions, the results could be considered reliable.

The results of brain activation discussed above are supported by the FC group analysis performed on all subjects studied as summarized in Fig. 3. In Fig. 3(a), it was found that in the left hemisphere, both HS and TLEP exhibited different trends of positive linear relationship between the two slopes indicating different behaviors in responses and activities in the two regions. It can be contended that in the left hemisphere, for all HSs and TLEPs participating in this study, the rate of change of the response in PRE due to activity in SMG was greater than the rate of change of the response in SMG due to activity in PRE when the brain was at rest. The rate of change was greater in HS compared to TLEP as indicated by the slope values. In Fig. 3(b), it indicated that, in the right hemisphere, both groups of subjects demonstrated a similar trend of positive linear relationship between the two slopes but with different behaviors in responses and activities in the two regions. A smaller increase in M $\mathrm{RPRE}^{-}$ RSMG caused a larger increase in MRSMG-RPRE by a factor of two. Similar to the plots for the left hemisphere for all HSs and TLEPs participating in this study, the rate of change of the response in PRE due to activity in SMG was greater than the rate of change of the response in SMG due to activity in PRE when the brain was at rest. However, the rate of change in TLEP was similar to the rate of change in HS as indicated by the slope values.

The FC analysis performed on both groups of subjects revealed important information about brain asymmetry shown particularly by PRE and SMG. The difference (brain asymmetry) between HS and TLEP can be seen in the left hemisphere. In the right hemisphere, FC between PRE and SMG in HS and TLEP was equal.

There were several limitations in this study. First, a small number of subjects were recruited for this study. Larger sample sizes should provide more reliable results with greater precision and power but with limited cost, time and funds in this study. Despite the drawback, the 
preliminary results obtained were still valid. Secondly, the TLEPs recruited in this study were mainly from patients who had been treated with anti-epileptic drugs. This could affect brain activity and thus functional connectivity. It is suggested for future studies, to obtain more meaningful findings, patients could stop treatment 24 hours before scanning time to observe brain activation without drug effects. Third, the non-homogenous subjects in terms of the causes of TLE included in this study were due to limited number of cases obtained from in-patient data only. In future, it is suggested that only homogenous causes of TLE will be recruited as it will create more specific findings relevant to the group.

In conclusion, changes in lateralisation for both regions were evident in TLEP. Data (NOV) indicated that both HS and TLEP showed right lateralisation in the supramarginal gyrus. The precuneus was left lateralized in HS and almost equally bilateralised in TLEP. These were the main differences observed for the group. Effort to understand these mechanisms could lead to improved treatment strategies to prevent impairment of consciousness and improve the quality of life of people with epilepsy.

This study incorporated some data to the growing evidence to indicate that resting-state functional MR imaging can be potentially useful for pre-surgical mapping of eloquent cortices 28 in patients with temporal lobe epilepsy. However, there were a few limitations which included methodological differences between prior studies, small sample size and the presence of various reference techniques. Since resting state networks are task independent, thus making this approach substantially more widely applicable.

\section{CONFLICT OF INTEREST}

No conflict of interest

\section{ACKNOWLEDGEMENT}

This project was funded by the Fundamental Research Grant Scheme (FRGS/1/2015/SKK02/UNISZA/02/1) and has no conflict of interest. The authors would like to thank Hospital Universiti Sains Malaysia (HUSM), particularly Department of Radiology, HUSM and their respective staff for their valuable assistance and permission.

\section{REFERENCES}

1. Patel A,Biso F. Connectome - Scholarpedia. In: Neuro Anatomy, Temporal Lobne. NLM Pubreader; 2020:NBK519512. Accessed March 31, 2020. www.ncbi.nlm.nih.gov/books/NBK519512/

2. Yang Z, Choupan J, Reutens D, Hocking J. Lateralization of temporal lobe epilepsy based on resting-state functional magnetic resonance imaging and machine learning. Front Neurol. 2015;6(Aug):1-9. doi:10.3389/fneur.2015.00184

3. Illman NA, Butler CR, Souchay C, Moulin CJA. D ' ej `a Experiences in Temporal Lobe Epilepsy.

Hindawi. 2012;2012(539567):15. doi:10.1155/2012/539567

4. Zhao F, Kang H, You L, Rastogi P, Venkatesh D, Chandra M. Neuropsychological deficits in temporal lobe epilepsy: A comprehensive review. Ann Indian Acad Neurol. 2014;17(4):374. doi:10.4103/09722327.144003

5. Davoodi-bojd E, Elisevich K V, Health S, et al. TLE Lateralization using Whole Brain Structural Connectivity. Conf Proc IEEE Eng Med Biol Soc. 2016;1103. doi:10.1109/EMBC.2016.7590896.TLE

6. Massot-Tarrús A, White K, Mirsattari SM. Comparing the Wada Test and Functional MRI for the Presurgical Evaluation of Memory in Temporal Lobe Epilepsy. Curr Neurol Neurosci Rep. 2019;19(6). doi:10.1007/s11910-019-0945-8

7. Conradi N, Rosenberg F, Knake S, et al. Wada test results contribute to the prediction of change in verbal learning and verbal memory function after temporal lobe epilepsy surgery. Sci Rep. 2021;11(1):110. doi:10.1038/s41598-021-90376-3

8. Qadri S, Dave H, Das R, Alick-Lindstrom S. Beyond the Wada: An updated approach to pre-surgical language and memory testing. Epilepsy Res. 2021;174 (January):106673. doi:10.1016/ j.eplepsyres.2021.106673 
9. Limotai C, Mirsattari SM. Role of Functional MRI in Presurgical Evaluation of Memory Function in Temporal Lobe Epilepsy. 2012;2012(Figure 3). doi:10.1155/2012/687219

10. Nadkarni TN, Andreoli MJ, Nair VA, et al. Usage of fMRI for pre-surgical planning in brain tumor and vascular lesion patients: Task and statistical threshold effects on language lateralization. NeuroImage Clin. 2015;7:415-423. doi:10.1016/j.nicl.2014.12.014

11. Reyes A, Thesen T, Wang X, et al. Resting-state functional MRI distinguishes temporal lobe epilepsy subtypes. Epilepsia. Published online 2016. doi:10.1111/epi.13456

12. Goldstein, Sam JAN. Habit Reversal-Hemispheres of the Brain, Lateralization of. Encyclopedia of Child Behavior and Development. 2011th ed. (Sam Goldstein JAN, ed.). Springer, Boston, MA; 2011. doi:10.1007/978-0-38779061-9

13. Uno MA, Reader SDC, Mcu ARM, The T.

Downloaded from https://academic.oup.com/ gigascience/article-abstract/5/suppl_1/s13742-0160147-0-n/2965219 by UNIVERSITI SULTAN ZAINAL ABIDIN ( UNiSZA ) user on 17 January 2019 Downloaded from https://academic.oup.com/ gigascience/article-abstract/5/suppl. 2016;5(Suppl 1):14-15. doi:10.1186/s13742-016-0147-0

14. Rosazza C, Ghielmetti F, Minati L, et al. Preoperative language lateralization in temporal lobe epilepsy (TLE) predicts peri-ictal, pre- and postoperative language performance: An fMRI study. NeuroImage Clin. 2013;3:73-83. doi:10.1016/ j.nicl.2013.07.001

15. Chai WJ, Abd Hamid AI, Abdullah JM. Modulatory difference due to injury pattern in the moderate-TBI brain: Effective connectivity of working memory from preliminary findings. J Phys Conf Ser. 2020;1497 (1). doi:10.1088/1742-6596/1497/1/012007

16. Seri FAS, Abd Hamid AI, Abdullah JM, Idris Z, Omar H. Brain responses to high frequencies (270 $\mathrm{Hz}-480 \mathrm{~Hz}$ ) changes due to vibratory stimulation of human fingertips: An fMRI study. J Phys Conf Ser. 2020;1497(1). doi:10.1088/17426596/1497/1/012012

17. Cordes D, Haughton VM, Arfanakis K, et al. Frequencies Contributing to Functional Connectivity in the Cerebral Cortex in " Resting-state "” Data. 2001;(August):1326-1333.

18. Vanhaudenhuyse A, Demertzi A, Schabus M, et al. Two Distinct Neuronal Networks Mediate the Awareness of Environment and of Self. Published online 2010:570-578.

19. Othman EA, Yusoff AN, Mohamad M, Abdul Manan H, Abd Hamid AI, Giampietro V. Hemispheric Lateralization of Auditory Working Memory Regions During Stochastic Resonance: An fMRI Study. J Magn Reson Imaging. 2020;51(6):18211828. doi:10.1002/jmri.27016

20. Jm S, Mar H, Hamid AIA, Ar M. Evaluation of default mode network in temporal lobe epilepsy patients and healthy subjects : a preliminary result. IOP Conf Ser Mater Sci Eng. Published online 2020. doi:10.1088/1757-899X/785/1/012047

21. Maldjian JA, Laurienti PJ, Kraft RA, Burdette JH. An automated method for neuroanatomic and cytoarchitectonic atlas-based interrogation of fMRI data sets. Neuroimage. 2003;19:1233-1239. doi:10.1016/S1053-8119(03)00169-1

22. Petsas N, Tinelli E, Lenzi D, et al. Evidence of Impaired Brain Activity Balance after Passive Sensorimotor Stimulation in Multiple Sclerosis. PLoS One. 2013;8(6):1-10. doi:10.1371/ journal.pone.0065315

23. Thibes RB, Novaes NP, Lucato LT, et al. Altered Functional Connectivity between Precuneus and Motor Systems in Parkinson's Disease Patients. Brain Connect. 2017;7(10):643-647. doi:10.1089/ brain.2017.0534

24. Mohd H, Rebuan A, Mohamed SJ, et al. Effective connectivity between precuneus and supramarginal gyrus in healthy subjects and temporal lobe epileptic patients. Med J Malaysia. 2021;76(3):360-368.

25. Nawi M, Rahmad, Hamid A, et al. Effective Connectivity of a Default Mode Network In Human Brain: In Search of a Dominant Node Using Spectral Dynamic Causal Modeling. Phys Technol Med Publ Malaysian Assoc Med Phys. 2020;1(1):1-14. http:/ / myjms.moe.gov.my/index.php/ptm\%0APhys. Technol. Med.\%0AEffective

26. Gris D. Resting State functional connectivity predits the strength of hemispheric lateralization for 
language prosessing in Temporal Lobe Epilepsy and control. Public Access NIH Public Access. 2013;185

(2):974-981. doi:10.1038/mp.2011.182.doi

27. Dietz A, Vannest J, Maloney T, Altaye M, Szaflarski JP, Holland SK. The Calculation of Language Lateralization Indices in Post-stroke Aphasia: A Comparison of a Standard and a Lesion-Adjusted Formula. Front Hum Neurosci. 2016;10(October):1-15. doi:10.3389/fnhum.2016.00493

28. Mitchell TJ, Hacker CD, Breshears JD. A Novel Data -Driven Approach to Preoperative Mapping of Functional Cortex Using Resting-State Functional Magnetic Resonance Imaging. Neurosurgery. 2013;73 (6):969-983. doi:doi: 10.1227/

NEU.0000000000000141. 